\title{
CEREBRAL EDEMA ASSOCIATED TO SCORPION STING: A TWO-CASE STING REPORT
}

\author{
ROMERO N. O. (1), HERNÁNDEZ T. J. M. (1)
}

(1) Hospital Del Niño Morelense, Cuernavaca, Morelos, Mexico.

ABSTRACT: Scorpionism is a public health problem in some places in Mexico. The clinical symptoms of envenomation by scorpion sting are by sympathetic and parasympathetic stimulation, developing systemic and local symptoms. The Central Nervous System (CNS) is one of the organs that are affected. In some cases, cerebral edema develops. In this report we present two pediatric cases with the association of envenomation by scorpion sting and cerebral edema. The first case developed severe cerebral edema, which progressed to a fatal outcome; and the other case developed mild cerebral edema with a satisfactory evolution. The pathophysiology of this complication is not well known and probably is the consequence of hypoxia, secondary to respiratory failure, laryngospasm and seizures that are manifestations of envenomation by scorpion sting.

KEY WORDS: cerebral edema, scorpion sting.

\section{CORRESPONDENCE TO:}

NEYDI OSNAYA ROMERO, Hospital Del Niño Morelense, Gustavo Gómez Azcarate, 205, P.O. BOX: 62270, Col. Lomas de la Selva, Cuernavaca, Morelos, México.

Fax: (525) 56069738. Email: nenyos@prodigy.net.mx. 


\section{INTRODUCTION}

Scorpionism is an important public health problem in Mexico due to the great incidence of scorpion stings in several states of the country. Scorpions are arthropods belonging to the arachnid class. One of the most toxic is the Centruroides limpidus limpidus, which is mainly found in the state of Morelos, Mexico (4).

Scorpion toxins are species-specific. They are composed of a biochemical complex of protein neurotoxins. It is believed that the binding capacity of toxins to specific membranes is due to their positively charged channels (9).

Clinical manifestations of envenomation by scorpion stings are believed to be due to a complex interaction between sympathetic and parasympathetic stimulation (1). The predominant mechanism of the scorpion toxin action depends on the particular species, as well as on the amount of toxin inoculated (10).

Local symptoms of envenomation by scorpion sting start seconds or minutes after inoculation at the affected site. The systemic symptoms develop 45 to 60 minutes after the sting and include CNS manifestations such as irritability, anxiety, hyperthermia, excitability, agitation, visual changes, nausea, vomiting, nystagmus, hyperreflexia, ataxia, hemiplegia, focal or generalized seizures, and encephalopathy (3). The scorpion toxin poorly passes through the blood-brain barrier and the effects on the CNS are secondary to the direct stimulation on the medullary sympathetic center (10). There is a sudden rise in blood pressure due to sympathetic stimulation, which can cause rupture of blood vessels, intracranial hemorrhage, encephalic infarcts, and failure of the respiratory center (7).

There are certain factors that may contribute to the development of neurological complications, such as cerebral edema. At the Children's Hospital of Morelos, we see approximately 30 cases a month, of which 10 (30\%) develop severe symptoms (8). Among those symptoms that place the patient's life at risk are: acute pulmonary and cerebral edemas. This is a two-case report of cerebral edema associated to scorpion sting.

\section{CASE 1}

Female patient, 3 years old, from a rural area in the state of Morelos, Mexico. Previously known to be healthy, she was seen at the General Hospital in Jojutla, Morelos, due to a scorpion sting occurring 11 hours previous to admission. The initial symptoms were local pain, irritability, excessive salivation, and nystagmus. Initial 
management included atropine, acetaminophen, hydrocortisone, and a dose of antiscorpion serum. The patient was transferred to another clinic where she had shortness of breath, was hyperthermic $\left(40^{\circ} \mathrm{C}\right)$, with trismus and nystagmus. Calcium gluconate (200-mg dose) was administered. Generalized seizures began and were treated.

Follow-up indications included: a second dose of anti-scorpion serum, hydrocortisone, and metamizol. No improvement was achieved, reason why she was transferred to the hospital's intensive care unit 24 hours after her admission to the General Hospital in Jojutla. The child had a fever $\left(40^{\circ} \mathrm{C}\right)$, was reported to have suffered generalized seizures, somnolence, isochoric pupils with a tendency to mydriasis, well-ventilated lungs, tachycardia, diminished abdominal peristalsis, and augmented osteotendon reflexes. The patient received hydration solutions parenterally (1500 $\mathrm{ml} / \mathrm{m}^{2}$ of her corporal surface [cs] - potassium, $40 \mathrm{mEq} / \mathrm{m}^{2} \mathrm{sc}$; calcium gluconate, $3 \mathrm{~g}$ - per day). Laboratory tests on admission reported: $\mathrm{Hb}, 11.8$; hematocrit, 37.5; leukocytes, 16,800; lymphocytes, 13\%; segments, 86\%; platelets, 171,000; calcium, $11.1 \mathrm{mEq} / \mathrm{ml}$.

The patient received diphenylhydantoin and a maintenance dose of $7 \mathrm{mg} / \mathrm{kg} / \mathrm{day}$, as well as Ranitidine, $2 \mathrm{mg} / \mathrm{kg} / \mathrm{day}$. Irritability and somnolence persisted; a lumbar puncture was performed to rule out any neurological infection. On the $3^{\text {rd }}$ day, the child deteriorated neurologically. She was seen by the neurology staff, who suggested a Computerized Axial Tomography (CAT scan) of the cranium, where a severe cerebral edema was confirmed (Figure 1). Dexamethasone and mannitol administration was initiated. On the $5^{\text {th }}$ day, generalized seizures started again, as well as vomiting of gastric contents, cyanosis, and bradycardia. After Cardiopulmonary Resuscitation (CPR) maneuvers, atropine was given again, orotracheal intubation was performed, and an electroencephalogram (EEG) was obtained, registering low voltage activity, presence of focal epilepsy, and multiple erratic discharges in the right hemisphere. Phenobarbital was added and due to hemodynamic instability, dobutamine was also administered. On the $7^{\text {th }}$ day, the patient presented suspected diabetes insipidus and cerebral death, and by the $10^{\text {th }}$ day, she was declared brain-dead, confirmed by an EEG. Later on, an irreversible cardiopulmonary arrest occurred. 


\section{CASE 2}

Female patient, 6 years old, without an important history related to her current condition. The current problem began 24 hours previous to admission, after being stung by a scorpion at home. Thirty minutes later, irritability and excessive salivation appeared, reason why she was taken to a physician. Hydrocortisone (two applications of $500 \mathrm{mg}$ each) and chlorpheniramine (two applications of $5 \mathrm{mg}$ each) were administered. The patient partially recovered and was taken home. Later, vomiting and intolerance appeared, and the patient returned to our hospital, 12 hours after the scorpion sting. The patient was admitted with dry oral mucosae, drowsiness, nystagmus, rales, no abdominal changes, and augmented osteotendon reflexes. Laboratory tests on admission included: sodium, $149 \mathrm{mEq}$; potassium, $2.89 \mathrm{mEq}$; and glucose, $108 \mathrm{mg} / \mathrm{dl}$

Parenteral hydration was initiated and 3 vials of anti-scorpion serum were administered at 30-minute intervals. Due to the persistent somnolence, a neurological assessment of the child's condition was sought. Clinical data suggested the child was suffering from cerebral edema. A CAT scan was ordered, confirming this diagnosis (Figure 2). Intravenous steroids were administered and the patient was discharged 48 hours later, asymptomatic and neurologically alert and intact.

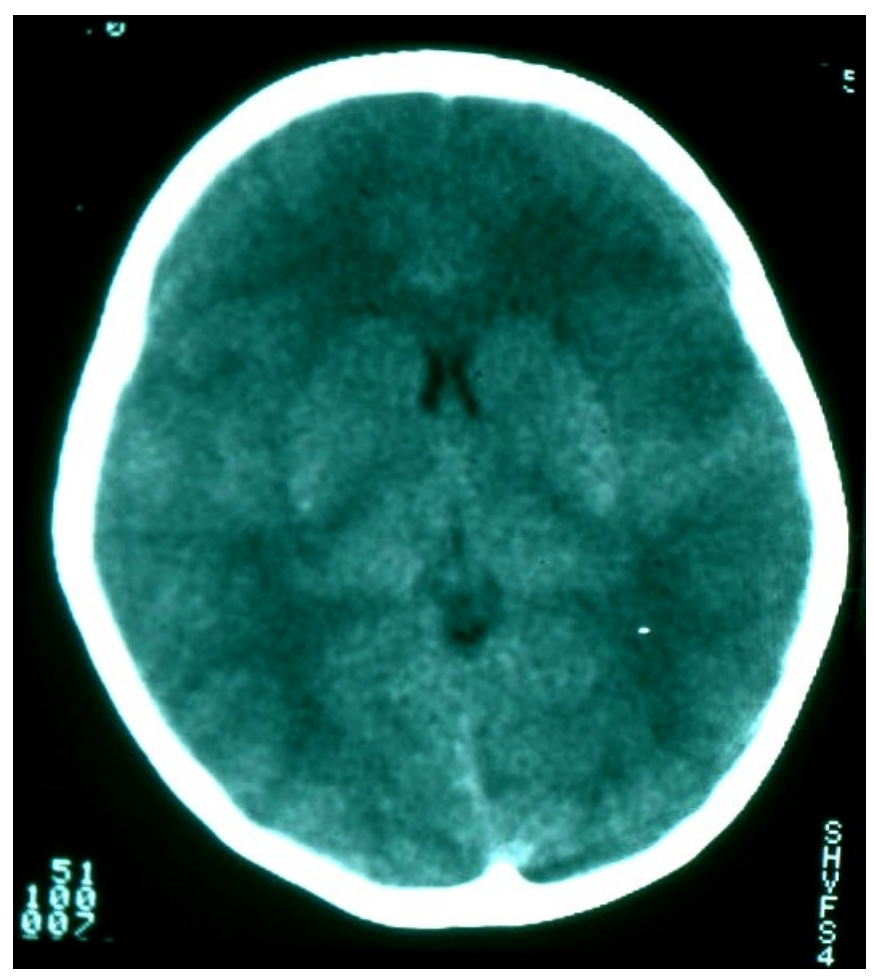

Figure 1: Computerized Axial Tomography (CAT) image of a severe cerebral edema in a child with severe scorpion envenomation. 


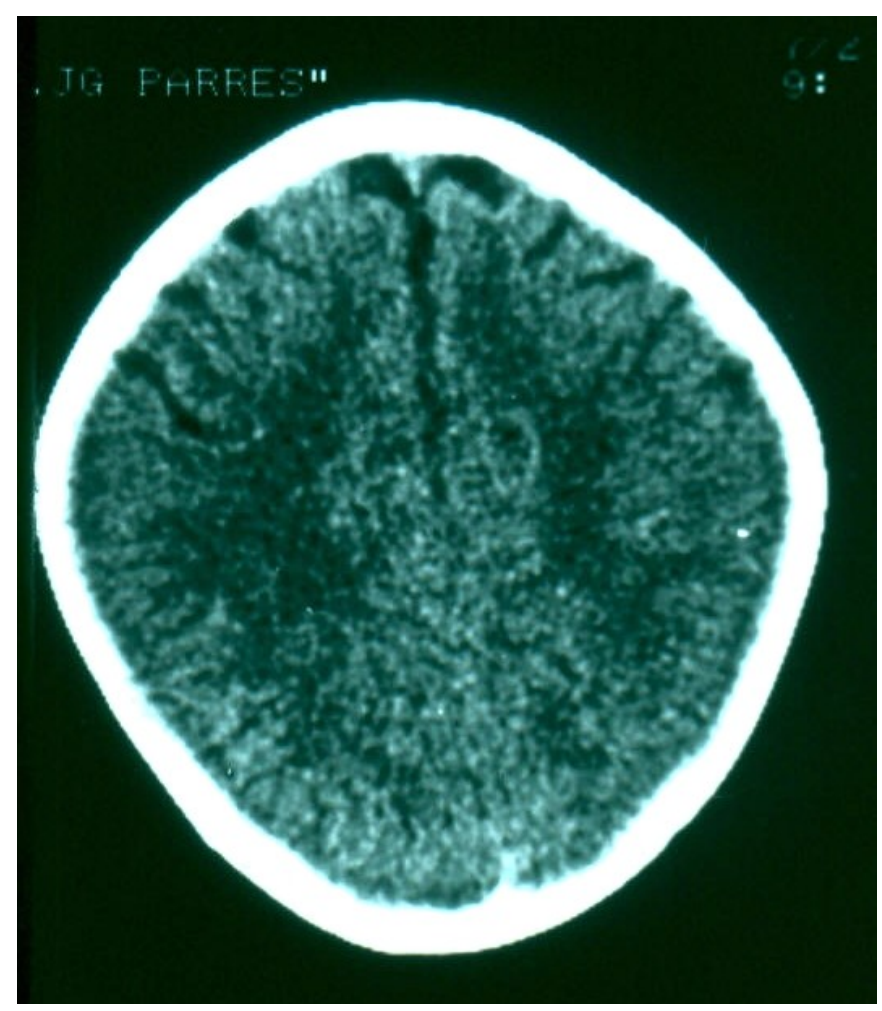

Figure 2: Computerized Axial Tomography (CAT) image of a mild cerebral edema in a child with severe scorpion envenomation.

\section{DISCUSSION}

CNS manifestations are infrequent in scorpion-stung patients, but nonetheless fatal, as seen in the first case. Many factors come into play in the pathophysiology of cerebral edema. Some of the protection measures are: adequate temperature control, good blood flow, which guarantees adequate oxygenation, and presence of substrata necessary for energy production. Hypercapnia may be present together with hypoxia, which in turn may be responsible for the accompanying neurological depression. Other causes are exogenous toxins that can directly damage the neuronal function. The metabolic acidosis/alkalosis itself can be enough to produce changes in the consciousness conditioning cerebral edema (5).

It has been reported that besides toxins deteriorate neuronal function directly, hypoxia, acidosis, enzymatic inhibition, hypoglycemia, or seizures may also have a contributing effect (5). The delivery of oxygen to the brain can be affected by changes that compromise the patient's airway, as may be the case of a laryngospasm (7). Neurons are very sensitive to the lack of oxygen and stop functioning seconds after initial oxygen deprivation (5). 
In cases of envenomation by scorpion sting, the effects on the CNS are unclear. It is known that the penetration of the toxin through the blood-brain barrier is poor, however certain authors agree the toxin acts directly on the neuron, producing encephalopathy and contributing to the occurrence of seizures. An increase in blood pressure secondary to sympathetic stimulation is also mentioned, which could cause hemorrhages, brain edema, and cerebral infarction $(2,6,7)$.

The presence of local symptomatology seems to occur almost immediately, while systemic symptomatology occurs within an hour after the accident. For this reason, it is necessary that the patient receive medical attention as soon as possible, together with an adequate treatment to avoid cerebral edema.

The first patient was seen 11 hours after the accident, while in the second case, even though the patient had systemic symptomatology, the child was seen 30 minutes after the accident. Symptomatic treatment was administered, favoring the absence of further complications. Both patients presented data of severe intoxication, one with seizures that alone could have conditioned hypoxia and favored the presence of severe cerebral edema, or the sympathetic stimulation conditioned a decrease in an adequate amount of oxygen.

Their laboratory tests showed leukocytosis, and in one, hypokalemia, for which no specific treatment was administered. We have detected that patients with severe symptomatology have metabolic acidosis, hypokalemia, and changes in the body temperature leading to negative inotropic factors for having a good source of oxygen. In cases of late or inadequate management, there is the possibility of complications that place our patients' lives at risk.

\section{REFERENCES}

1 AMITAI Y. Clinical manifestations and management of scorpion envenomation. Public Health Rev., 1998, 26, 257-63.

2 BONILHA L., CENDES F., GHIZONI E., VIEIRA RJ. Epilepsy due to a destructive brain lesion caused by a scorpion sting. Arch. Neurol., 2004, 61, 1294-6.

3 DEHESA DM., POSANNI LD. Scorpionism and serotherapy in Mexico. Toxicon, 1994, 32, 1015-8.

4 DÍAZ NA. Listas y datos de distribución geográfica de los alacranes de México. Rev. Inv. Salud Publica, 1975, 35, 1-36. 
N. O. Romero and T. J. M. Hernández CEREBRAL EDEMA ASSOCIATED TO SCORPION STING: A TWOCASE STING REPORT. J. Venom. Anim. Toxins incl. Trop. Dis., 2005, 11, 4, p. 600

5 DOUGLAS NMD. Coma and altered level of consciousness. In: FLEISHER GR., LUDWING S. Textbook of Pediatric Emergency. 4.ed. Philadelphia: Lippincott Williams \& Wilkins, 2000: 165-8.

6 ESCORZA IC., DE LEÓN B. Picadura de Alacrán. Análisis de un caso de consecuencias fatales. Acta Pediatr. Mex., 1996, 17, 150-6.

7 MAHADEVAN S. Scorpion sting. Indian Pediatr., 2000, 37, 504-14.

8 OSNAYA RN., MEDINA HT., HERNÁNDEZ FS., LEÓN RG. Clinical symptoms observed in children envenomated by scorpion stings, at the children's hospital from the State of Morelos, Mexico. Toxicon, 2001, 39, 781-5.

9 POSSANI LD. Structure of scorpion toxins. In : TU AT. Ed. Handbook of Natural Toxins. New York: Marcel Dekker, 1984: 513-50.

10 REEVES JJ. Scorpion envenomation. Clin. Toxicol. Rev., 1998, 20, 1-6. 